\title{
Generation, analysis and functional annotation of expressed sequence tags from the ectoparasitic mite Psoroptes ovis
}

\author{
Stewart TG Burgess ${ }^{*}$, Alasdair J Nisbet, Fiona Kenyon and John F Huntley
}

\begin{abstract}
Background: Sheep scab is caused by Psoroptes ovis and is arguably the most important ectoparasitic disease affecting sheep in the UK. The disease is highly contagious and causes and considerable pruritis and irritation and is therefore a major welfare concern. Current methods of treatment are unsustainable and in order to elucidate novel methods of disease control a more comprehensive understanding of the parasite is required. To date, no full genomic DNA sequence or large scale transcript datasets are available and prior to this study only 484 P. ovis expressed sequence tags (ESTs) were accessible in public databases.

Results: In order to further expand upon the transcriptomic coverage of $P$. ovis thus facilitating novel insights into the mite biology we undertook a larger scale EST approach, incorporating newly generated and previously described P. ovis transcript data and representing the largest collection of P. ovis ESTs to date. We sequenced 1,574 ESTs and assembled these along with 484 previously generated $P$. ovis ESTs, which resulted in the identification of 1,545 unique $P$. ovis sequences. BLASTX searches identified 961 ESTs with significant hits (E-value < 1E-04) and 584 novel P. ovis ESTs. Gene Ontology (GO) analysis allowed the functional annotation of 880 ESTs and included predictions of signal peptide and transmembrane domains; allowing the identification of potential $P$. ovis excreted/ secreted factors, and mapping of metabolic pathways.

Conclusions: This dataset currently represents the largest collection of P. ovis ESTs, all of which are publicly available in the GenBank EST database (dbEST) (accession numbers FR748230 - FR749648). Functional analysis of this dataset identified important homologues, including house dust mite allergens and tick salivary factors. These findings offer new insights into the underlying biology of $P$. ovis, facilitating further investigations into mite biology and the identification of novel methods of intervention.
\end{abstract}

\section{Background}

Sheep scab, caused by the mite Psoroptes ovis is, arguably, the most important ectoparasitic disease of sheep in the UK in terms of both animal welfare and financial impact. In continental Europe and in the USA, psoroptic mange is also a welfare problem in cattle and is becoming increasingly common in cattle in the UK [1]. Psoroptic mange is highly contagious, causing considerable pruritis and irritation [2]. Current disease control strategies are heavily reliant upon chemotherapy; however concerns over residues, eco-toxicity and the development of parasite resistance threaten the sustainability of

\footnotetext{
*Correspondence: stewart.burgess@moredun.ac.uk
Division of Parasitology, Moredun Research Institute, Pentlands Science Park,

* Correspondence: stewart.burgess@moredun.ac.uk
Division of Parasitology, Moredun Research Institute, Pentlands Science Park, Bush Loan, Edinburgh, UK
}

(c) 2011 Burgess et al; licensee BioMed Central Ltd. This is an Open Access article distributed under the terms of the Creative Commons Attribution License (http://creativecommons.org/licenses/by/2.0), which permits unrestricted use, distribution, and reproduction in any medium, provided the original work is properly cited. Such approaches require a more comprehensive understanding of both the parasite and its interaction with the host.

P. ovis is a non-burrowing, surface exudate feeder capable of consuming serous fluids, lymph and red blood cells [4]. Mites survive on the surface of the skin and do not appear to penetrate beyond the stratum corneum, the outermost layer of the skin [5]. The available evidence suggests that mites abrade the stratum corneum and deposit allergens as they progress - the combination of mechanical skin abrasion, mite allergen deposition and increased self-grooming behaviour by the host in response to the pruritis caused by the mites triggers the in the development of alternative control methods [3] 
subsequent activation of a cutaneous inflammatory response [6,7] providing an exudate which supplies the mite with a food source [8]. Establishment of a P. ovis infestation is therefore the result of a complex interaction between the host and the mite, during which the mite appears to initiate reactions conducive to its own establishment and maintenance [9]. The skin lesions are induced by mite-derived pro-inflammatory factors, a likely source of which is mite excretory/secretory products, including potent enzymes and allergens (reviewed in [8]). While several sheep scab mite products have been identified, including a number of enzymes and homologues of allergens of the house dust mite (HDM), Dermatophagoides pteronyssinus and the scabies mite Sarcoptes scabiei, their functions remain largely unknown [10].

Prior infestation with sheep scab mites alters the progression of subsequent infestations and reductions in lesion size have been observed in secondary infestations in sheep, suggesting that vaccination against the parasite may be feasible [11]. Vaccination with $P$. ovis extracts has resulted in a 15-fold reduction in mite numbers and a 4-fold reduction in lesion size [12,13]. However, identification of the individual proteins involved in protective immunity has not yet been achieved. The lack of available $P$. ovis sequence information is hampering vaccine candidate discovery, whether that process is through the pragmatic or rational routes. To date, no full genomic DNA sequence has been available for $P$. ovis and this is the case for most related mite species, i. e. Dermatophagoides pteronyssinus and Sarcoptes scabiei, where the main focus has been on the generation and analysis of expressed sequence tags (ESTs). To date, only 484 P. ovis ESTs have been available in the public databases [14] and, in order to further our understanding of the biology of $P$. ovis, we have now generated a complementary DNA (cDNA) library and have undertaken a larger scale EST approach and incorporated these newly generated and previously-described $P$. ovis ESTs with new bioinformatic analyses to identify further novel mite-expressed genes. This paper describes this approach, which represents the largest such resource for $P$. ovis, along with the functional annotation of the ESTs to further our understanding of this economically important parasite.

\section{Results and Discussion CDNA library and EST analysis}

1574 ESTs were derived from a normalised cDNA library constructed from a pool of mixed life stages and mixed sex mites (Library 1). To this dataset we added a further $484 \mathrm{P}$. ovis ESTs previously generated at the Moredun Research Institute (Library 2) [14], giving a total of 2,058 ESTs (Table 1). Filtering for low quality
Table $1 P$. ovis EST analysis and breakdown of processing stages

\begin{tabular}{cc}
\hline Sequence Description & Number of ESTs \\
\hline CDNA Library 1 & 1574 \\
CDNA Library 2 & 484 \\
Total ESTs & $\mathbf{2 0 5 8}$ \\
Removal of low quality sequences & -153 \\
Total remaining ESTs & $\mathbf{1 9 0 5}$ \\
CAP3 Contigs & 255 \\
Singletons & 1290 \\
Total ESTs & $\mathbf{1 5 4 5}$ \\
Significant BLAST hits & 961 \\
No or non-significant BLAST hit & 584
\end{tabular}

Summary of $P$. ovis EST processing steps and results of BLAST search.

sequences resulted in the removal of 153 ESTs, leaving 1905 sequences for assembly of contigs (93\% sequencing efficiency). CAP3 contig assembly resulted in the identification of 255 contiguous sequences (consisting of 2 or more ESTs) and 1290 singletons giving a total of 1545 unique sequences (Table 1). Overall sequence length including both contigs and singletons ranged from $187 \mathrm{bp}-1553 \mathrm{bp}$, with a mean length of $700 \mathrm{bp}$ (contigs: mean length $=800 \mathrm{bp}$, range $=397 \mathrm{bp}-1553 \mathrm{bp}$; singletons: mean length $=675 \mathrm{bp}$, range $=187 \mathrm{bp}-922$ bp). All new ESTs generated here were submitted to the EST database (dbEST) at GenBank under the accession numbers FR748230 - FR749648. BLAST analysis of the 1545 assembled ESTs identified 961 (62\%) with significant BLAST hits. Of these, 192 represented contiguous sequences, whilst the remaining 769 were singletons; summary details of the largest contigs (Top 10) are described in Table 2. E-values for the 961 ESTs ranged from 1.0E-4 to $3.6 \mathrm{E}-180$. The mean percentage similarity score between and EST and its closest homologue was $68.4 \%$, ranging from $36 \%-100 \%$. The most abundant phylum associated with the top BLAST hits was that of the arthropoda, including insects, i.e. Drosophila melanogaster, a number of ticks, i.e. Ixodes scapularis (deer tick) and other mites, i.e. Blomia tropicalis, D. pteronyssinus and $D$. farinae. The top BLAST hit species was $I$. scapularis which represents the most closely related organism to $P$. ovis for which a fully sequenced genome exists. The remaining 584 ESTs with either no BLAST hit or non-significant hits may represent novel P. ovis transcripts, of as yet undefined function.

\section{Functional annotation of $P$. ovis ESTs \\ Gene Ontology (GO) term annotation and mapping}

In order to gain a functional understanding of the assembled ESTs Gene Ontology (GO) annotation was associated with each of the sequences within the blast2go application [15-17]. This process involved mapping 
Table 2 Details of the largest contigs from the $\boldsymbol{P}$. ovis EST dataset

\begin{tabular}{ccccc}
\hline Contig ID & Contig Size (bp) & No. of ESTs represented & Example EST ID from Contig & Top Blast hit \\
\hline Contig 63 & 1553 & 2 & Bu_007_c09 & Karyopherin alpha 6 \\
Contig 95 & 1529 & 4 & Bu_012_f01 & GTP-binding protein SAR1B \\
Contig 28 & 1520 & 4 & Bu_004_g08 & T-complex protein 1 subunit gamma \\
Contig 195 & 1481 & 4 & Bu_003_a06 & Mitochondrial ribosomal protein 143 \\
Contig 78 & 1435 & 5 & Bu_010_d06 & Chitinase \\
Contig 246 & 1322 & 2 & Bu_010_a01 & WD repeat and FYVE domain containing 2 \\
Contig 107 & 1310 & 3 & Bu_013_c11 & RAS-related GTP binding A \\
Contig 236 & 1297 & 3 & Bu_011_d04 & Hypothetical protein ISCW014890 (Ixodes scapularis) \\
Contig 71 & 1226 & 2 & Bu_009_g11 & NIPSNAP1 protein \\
Contig 121 & 1201 & Bu_014_f12 & Sphingomyelinase D-like protein \\
\hline
\end{tabular}

Summary details of the top 10 largest contigs from the $P$. ovis EST dataset. Example EST ID refers to the individual EST ID from the $P$. ovis EST dataset.

the BLAST hits against known GO terms and then further annotation of the selected GO terms by integrating data from a range of sources including; InterPro to derive protein families and domains; Enzyme Commission (EC) codes to determine distinct functional enzyme class data and Kyoto Encylopedia of Genes and Genomes (KEGG) to identify enriched signalling pathways. Of the 961 ESTs with significant BLAST hits, 880 were associated with GO terms. The majority of sequences were associated with more than one GO term $(n=743)$, with some being associated with as many as 50 individual GO terms. Only 81 ESTs (8.4\%) could not be mapped to a particular GO annotation. The GO terms were split over the 3 main categories [Biological Process (BP), Molecular Function (MF) and Cellular Component (CC)] with the majority in the BP and MF categories. In order to visualise the GO terms represented by the ESTs we mapped the second level GO terms across all three categories (BP, MF and $\mathrm{CC}$ ) and these are presented as percentage pie-charts in Figure 1. In the BP category the majority of second level GO terms were associated with either cellular $(23 \%)$ or metabolic processes (20\%), in the MF category the majority of GO terms were associated with either catalytic activity (37\%) or binding processes (45\%) and in the CC category most were associated either with the cell $(43 \%)$ or organelle (31\%). Also of note in the BP category were terms associated with locomotion, reproduction, growth, biological regulation, developmental process, immune system process and response to stimulus. In the MF category a number of sequences were found to be associated with enzyme and transcriptional regulator activity and molecular transducer activity. A multi-level GO term analysis provided further insights into EST function, revealing the nature of the binding and catalytic activities highlighted at the second level of GO terms in the MF category. A number of enzyme classes were identified including protein kinases, ligases, oxidoreductases, hydrolases and endopeptidases. Amongst the binding activities identified were ATP, GTP, RNA and DNA binding, as well as zinc ion binding, co-factor binding and cytoskeletal protein binding. Multi-level GO term analysis of the BP category revealed a number of interesting associations, of particular note were reproductive developmental processes, signalling pathways, cell development, cytoskeleton organisation, nervous system development, proteolysis and response to stress and chemical stimulus. Multi-level analysis of the CC category revealed a number of sequences with terms associated with the cytosol, nucleoplasm, cytoskeleton, plasma membrane, lipid particles and integral membrane molecules.

\section{Identification of excreted/secreted proteins}

Interpro consists of a collection of protein family and domain signatures and can be used for the automatic annotation of proteins [18]. The Interproscan software tool allows the scanning of protein sequences against the Interpro database using a range of profile-based hidden Markov models ( $\mathrm{pHMM}$ ) and positional specific score matrix protocols and in this way overcomes the problems inherent in single database searches [19]. Of the 961 ESTs with significant BLAST hits, Interpro was able to map 849 , leaving 112 with no further protein domain mapping. 533 ESTs were found with both GO term mapping and Interpro mapping, providing both gene onotology and protein domain information, whilst 316 ESTs were successfully mapped by Interpro but not to a specific GO. Interproscan incorporates searches for structural and functional annotation of protein sequences, along with prediction of signal peptide sequences (SignalP) and transmembrane helices (TMHMM) [18,20,21]. Potential secretory proteins, secreted through the classical pathway, can be identified through the presence of a signal peptide sequence [22]. This sequence targets proteins for translocation across the ER membrane and is an $\mathrm{N}$-terminal peptide, typically 15-30 amino acids long which is cleaved during translocation [22]. Not all proteins with SPs are secreted 


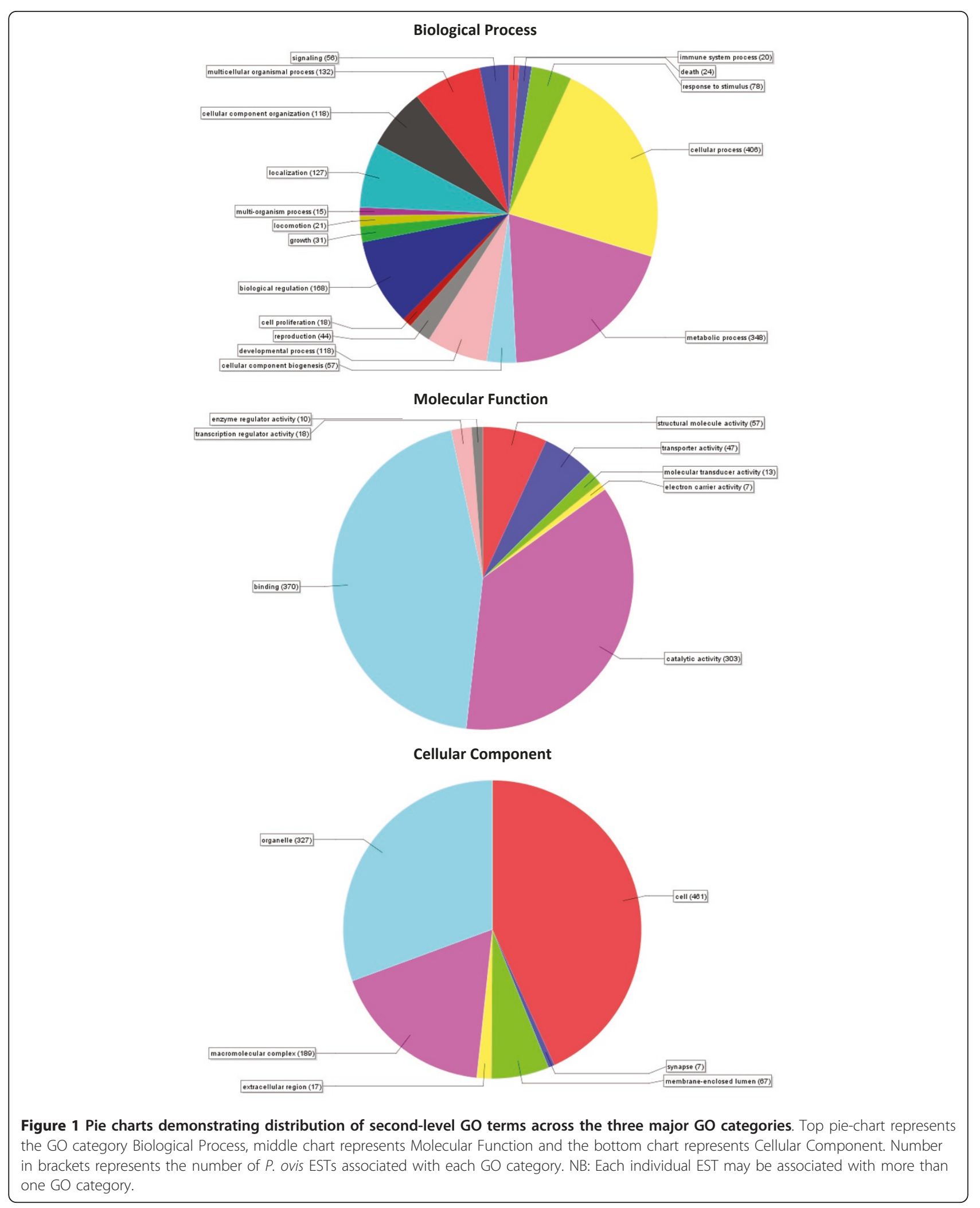


and some may become integrated into the plasma membrane, i.e. transmembrane [22]. This type of profiling can help to identify potentially secreted/excreted peptides and can aid in the identification of signalling pathways and potential diagnostic or therapeutic targets [20]. Interproscan revealed the presence of 173 ESTs with predicted signal peptides (SP) and 191 sequences with a predicted transmembrane domain (TMD). By filtering the SP sequences against the TMD sequences we were able to identify 74 ESTs with a predicted SP but no TMD, thus representing potential P. ovis excretory/ secretory proteins. A further 183 ESTs were identified with predicted SP and TMDs along with a predicted glycophosphatidylinositol (GPI) anchor sequence thus representing potential $P$. ovis excreted/secreted outer membrane proteins. A final group of 129 ESTs was identified with no predicted SP but with predicted TMD and GPI anchor sequence and this group may represent $P$. ovis outer membrane proteins. The identification of a number of $P$. ovis sequences predicted to encode either secreted or membrane integrated proteins is an important step in the elucidation of mite factors as potential therapeutic targets. By their nature, secreted and/or membrane bound factors are more likely than cytosolic factors to be encountered by the host immune system and may therefore represent valid therapeutic or diagnostic targets. As $P$. ovis is a non-burrowing mite it is thought to rely on the action of a number of proteases to trigger a host inflammatory response to produce an inflammatory exudate, upon which it is then able to feed [8]. It is highly likely that some of these proteases are either secreted or excreted, for example the HDM protein Der p 2 (of which Pso o 2 is a homologue) has been shown to be derived from cells which line the mite gut and has been found in mite faecal pellets [23]. Further interrogation of these ESTs may lead to the identification of novel $P$. ovis E/S factors and therefore new therapeutic or diagnostic targets.

\section{Enzyme code annotation}

Of the 961 ESTs with significant BLAST hits, 223 (23\%) mapped to a specific Enzyme Commission (EC) code. Of these, 126 mapped to unique EC codes and a number of enzyme classes were represented, including oxidoreductases $(\mathrm{n}=52)$, transferases $(\mathrm{n}=50)$, hydrolases $(\mathrm{n}=99)$, lysases $(\mathrm{n}=4)$, isomerases $(\mathrm{n}=5)$ and ligases $(\mathrm{n}=13)$ (Table 3$)$. Thirty nine ESTs mapped to a single EC code, 3.6.5.3, which represents protein-synthesising GTPases. These enzymes are involved in mRNA translation into protein by the ribosome and include translation initiation factors. This process is involved in the regulation of gene expression by controlling translation rates and therefore could be involved in a number of processes including the control of stage-specific gene expression in $P$. ovis [24]. A number of $P$. ovis enzyme activities have previously been identified; most of these are proteolytic and involve cysteine, metalloproteinases and aspartyl proteases [25]. These enzymes are able to digest a number of key protein substrates, including fibronectin, haemoglobin, and fibrinogen and may be involved in mite feeding [25]. Amongst the ESTs analysed here we have confirmed the presence of these enzyme classes and also identified transcripts encoding a number of additional proteases, namely dipeptidase (n $=1$ ), aminopeptidase $(\mathrm{n}=1)$, serine-type carboxypeptidases $(n=3)$, metallocarboxypeptidase $(n=1)$, metalloendopeptidases $(n=2)$, threonine endopeptidases $(n=$ 6) and two further proteases (nedd 88 and a prolyl endopeptidase). The cathepsins identified here represent $P$. ovis cysteine proteases and may have roles in mite digestive processes as has been shown for a cathepsin L-like molecule potentially involved in meal digestion in the cattle tick Rhipicephalus microplus [26]. Digestive system-derived proteases are likely to be important in food processing in the mite and, as with the aspartyl proteinase Na-APR-1 of hookworms, may therefore represent highly effective vaccine candidates. In addition, mite derived cysteine proteinase molecules may also interact with host keratinocyte-derived cystatin-A, which is a cysteine protease inhibitor produced as part of the host cutaneous immune response to mite-derived allergens [27].

Additional EC classes identified included thioredoxins, glutathione S transferases (GSTs), peroxiredoxins and cyclophilins (immunophilins). Thioredoxins and peroxiredoxins are antioxidant enzymes which could form a part of the mites defence against reactive oxygen species (ROS) generated during the host inflammatory response. Homologous enzymes have been identified in HDMs and have been implicated in the scavenging of ROS and depression of superoxide dismutase, glutathione peroxidise and catalase activities [28,29].

A number of GSTs were identified from the $P$. ovis ESTs described here and these include delta, mu and alpha classes. GSTs catalyse a variety of enzymatic reactions but perhaps the most relevant is the detoxification and breakdown of xenobiotic substances and these enzymes may be involved in the mites' defence against a range of environmental and host derived compounds. For example, increased transcription of mu, and delta class GSTs has been demonstrated in S. scabiei mites following exposure to the acaricidal compound permethrin [30]. GSTs have also been shown to react strongly with host IgE and IgG suggesting a further role in the development of the allergic immune response [31-33].

Two ESTs encoding scavenger receptor cysteine rich protein (SRCRP) and cysteine rich venom protein with trypsin inhibitor like (TIL) domains [34] were also identified. Secretion of proteins with trypsin inhibitory 
Table 3 Summary of EST dataset

\begin{tabular}{|c|c|c|c|c|c|c|}
\hline Gene name & $\begin{array}{l}\text { Putative function/ } \\
\text { enzyme class }\end{array}$ & $\begin{array}{l}\text { Homologous protein in } \\
\text { house dust mite }\end{array}$ & Top species hit & $\begin{array}{l}\text { BLASTX E- } \\
\text { value }\end{array}$ & $\begin{array}{c}\text { EST } \\
\text { length } \\
\text { (bp) }\end{array}$ & $\begin{array}{l}\text { Similarity } \\
(\%)\end{array}$ \\
\hline \multicolumn{7}{|l|}{ Enzymes } \\
\hline Cathepsin B & Cysteine protease & - & Ixodes scapularis & $5.9 \mathrm{E}-83$ & 1110 & 67 \\
\hline Cathepsin L & Cysteine protease & - & Suidasia medanensis & $6.9 \mathrm{E}-19$ & 541 & 53 \\
\hline Thioredoxin-1 & Thioredoxin & - & Eriocheir sinensis & $1.5 \mathrm{E}-21$ & 751 & 76 \\
\hline $\begin{array}{c}\text { Glutathione } S \text { transferase } \\
\text { delta class } 2\end{array}$ & $\begin{array}{l}\text { Glutathione-S- } \\
\text { transferase }\end{array}$ & - & $\begin{array}{l}\text { Dermatophagoides } \\
\text { pteronyssinus }\end{array}$ & $6.5 \mathrm{E}-45$ & 569 & 67 \\
\hline Peroxiredoxin-2 & Peroxiredoxin & - & I. scapularis & $1.4 \mathrm{E}-25$ & 771 & 85 \\
\hline $\begin{array}{l}\text { FK506-binding protein } \\
\text { (cyclophilin-like) }\end{array}$ & Cyclophilin & - & Drosophila persimilis & $5.1 \mathrm{E}-48$ & 646 & 90 \\
\hline \multicolumn{7}{|l|}{ Allergens } \\
\hline Pso o 1 & Cysteine protease & Der f 1/Der $p 1$ & D. farinae & $9.36 \mathrm{E}-90$ & 735 & 87 \\
\hline Pso o 2 & MD-2-like & Der $\mathrm{f}$ 2/Der $\mathrm{p} 2$ & D. farinae & $2.4 \mathrm{E}-77$ & 625 & 87 \\
\hline Pso o 3 & Serine protease & Der $f$ 3/Der $p 3$ & Euroglyphus maynei & $1.4 \mathrm{E}-62$ & 801 & 73 \\
\hline Pso o 5 & Unknown & Der f 5/Der p 5 & D. farinae & $5.3 \mathrm{E}-35$ & 598 & 80 \\
\hline Pso $\circ 7$ & Unknown & Der f 7/Der p 7 & D. farinae & $9.6 \mathrm{E}-41$ & 710 & 70 \\
\hline Pso o 13 & Unknown & Der p 13 & D. pteronyssinus & $1.1 \mathrm{E}-13$ & 712 & 60 \\
\hline Pso $\circ 21$ & Unknown & Der f 21/Der p 21 & D. farinae & $2.4 \mathrm{E}-36$ & 624 & 78 \\
\hline \multicolumn{7}{|l|}{ Tick homologues } \\
\hline Fed tick salivary protein 5 & $\begin{array}{l}\text { Small heat shock } \\
\text { protein }\end{array}$ & - & I. scapularis & $5.2 \mathrm{E}-14$ & 763 & 59 \\
\hline $\begin{array}{l}\text { Secreted salivary gland } \\
\text { protein }\end{array}$ & Secreted protein & - & I. scapularis & $1.3 \mathrm{E}-7$ & 679 & 47 \\
\hline Heat shock protein 20.6 & Heat shock protein & - & I. scapularis & 4.7E-64 & 1103 & 75 \\
\hline $\begin{array}{l}\text { Group } 10 \text { secretory } \\
\text { phospholipase A2 }\end{array}$ & Phospholipase & - & Nasonia vitripennis & $1.6 \mathrm{E}-31$ & 748 & 59 \\
\hline Chitinase & Chitinase & - & $\begin{array}{c}\text { Saccharopolyspora } \\
\text { erythraea }\end{array}$ & 5.7E-71 & 1435 & 54 \\
\hline $\begin{array}{l}\text { Peritrophic membrane chitin } \\
\text { binding protein }\end{array}$ & Chitin binding protein & - & $\begin{array}{c}\text { Culex } \\
\text { quinquefasciatus }\end{array}$ & 7.4E-59 & 1073 & 51 \\
\hline \multicolumn{7}{|l|}{ Others ESTs of interest } \\
\hline $\begin{array}{l}\text { Macrophage migration } \\
\text { inhibition factor }\end{array}$ & Lymphokine & - & $\begin{array}{l}\text { Amblyomma } \\
\text { americanum }\end{array}$ & $6.5 \mathrm{E}-21$ & 945 & 60 \\
\hline $\begin{array}{l}\text { Spermatogenesis associated } \\
\text { factor }\end{array}$ & Unknown & - & Anopheles gambiae & 1.1E-27 & 604 & 72 \\
\hline Cytochrome P450 & Oxidation & - & $\begin{array}{l}\text { Saccoglossus } \\
\text { kowalevskii }\end{array}$ & $1.7 \mathrm{E}-25$ & 659 & 60 \\
\hline
\end{tabular}

Table shows sequences encoding for proteins with homology to house dust mite allergens, tick proteins and enzymes of interest to $P$. ovis biology.

domains may increase mite survival by blocking host immune molecules or by interfering with proteolytic actions, as has been previously described for a TIL domain-containing peptide from the cattle tick Rhipicephalus microplus [35]. This factor may also regulate the actions of proteases secreted by the mites themselves. The physiology of the skin and the integrity of an effective skin barrier are maintained by the correct balance of protease and anti-protease activities [36] -the disruption of barrier function through the interruption of the fine balance in protease activity may represent one means by which mites achieve allergen infiltration and thus immune activation. The identification here of a number of additional enzyme classes from $P$. ovis has further expanded our current understanding of the enzymatic repertoire of this economically important ectoparasite and this knowledge will aid in the identification of novel means of targeting $P$. ovis and interfering with the host-parasite relationship.

\section{Allergen homologues}

A number of $P$. ovis antigens/allergens have previously been identified through their homology with molecules from other mite species, for example the HDM, D. pteronyssinus and the scabies mite S. scabiei $[37,38]$. Among these are homologues of the HDM allergens Der $\mathrm{p} 1$ (termed Pso o 1), Der p 2 (Pso o 2), Der p 5 (Pso-der p V-1), Der p 7 (Pso-gp7-1), Der p 10 (tropomyosin), Der p 11 (paramyosin) and Der p 14 (M177/apolipophorin/ 
vitellogenin) [25,39]. Whilst confirming the presence of these homologous genes in P. ovis, the EST analysis described here has also led to the identification of additional HDM homologues, namely Der p 21 (Pso o 21), Der p 3 (Pso o 3) and Der p 13 (Pso o 13) which may represent $P$. ovis allergens (Table 3 ). The action of a number of these allergens has been widely described in the literature for HDMs (for a review see [10]), however, homologues of Der p 3 and Der p 21 have not been previously described in $P$. ovis. Der p 21 is a $15 \mathrm{kDa}$ allergenic protein which is excreted in HDM faecal pellets and has been associated with allergic asthma [40]. HDM Der p 3 allergen is a $30 \mathrm{kDa}$ serine protease with trypsin-like activity that is able to enzymatically digest the complement components $\mathrm{C} 3$ and $\mathrm{C} 5$, generating the anaphylatoxins $\mathrm{C} 3 \mathrm{a}$ and $\mathrm{C} 5 \mathrm{a}$, thus contributing to the pathogenesis of allergic disease [41]. We have previously demonstrated the involvement of the complement system in the host response to P. ovis [6] and the identification of a Der $\mathrm{p} 3$ homologue in $P$. ovis adds further support to this finding. The identification of a Der $\mathrm{p} 21$ homologue is a significant finding and could indicate a novel mechanism by which $P$. ovis interacts with the host immune response. The confirmation of the presence of these highly conserved allergens across a number of mite species indicates that they are likely to perform functions that are crucial to mite biology and survival, which marks them out as prime targets for intervention.

\section{Tick homologues}

The species with the highest representative number of top BLAST hits was the deer tick Ixodes scapularis. In recent years a large number of ESTs have been recorded for this parasitic tick in public databases, and it probably represents the most studied, closely related species to the $P$. ovis. We identified a number of homologues of tick proteins including four homologues of tick secreted salivary gland proteins, ten tick heat shock proteins including a homologue of the fed tick salivary protein 5 (FTSP5), which has been shown to be upregulated in a number of invertebrate pathologies and may be involved in the response to hypoxia and oxidative stress [42,43], and a Group 10 secretory phospholipase A2 (PLA2G10) homologue (Table 3). Of particular interest is the presence of homologues of fed-tick and secreted salivary gland proteins, as the secretion of proteins and potential allergens by $P$. ovis has been postulated, but not proven to date, neither has the existence of a salivary gland or similar structure. The identification of $P$. ovis salivary proteins representing possible allergens is intriguing from a control perspective as their secretory nature makes it highly likely that these molecules would be seen by the host immune response. Further investigation of these factors may identify secretory/excretory pathways in $P$. ovis that could be exploited as diagnostic or therapeutic targets. Additional ESTs which may have a bearing on key aspects of mite biology included a homologue of a tick chitinase and other chitin molecules, one of which shared homology with a peritrophic membrane (PM) chitin binding protein. The PM is composed of chitins and proteins and forms a film-like structure which separates food from the midgut tissue in a number of arthropod species [44]. Therefore this PM molecule could potentially play a role in the formation of the mite gut digestive architecture. We also identified 4 homologues of tick cuticle proteins and it is likely that these are involved in the development of the mite exoskeleton.

\section{Additional ESTs of interest}

A number of other mite proteins that could be involved in the host-parasite interaction in sheep scab were also identified, including a homologue of macrophage migration inhibition factor (MIF) from the lone star tick Amblyomma americanum. In humans, MIF encodes a lymphokine involved in cell-mediated immunity and the regulation of inflammatory responses [45]. Homologues of MIF have been identified in a number of parasites including the malaria parasite Plasmodium falciparum [46] and are thought to play a role in manipulation of the host immune response to enhance parasite survival in a number of species of helminth parasites. A homologue of a schistosome major egg antigen was also identified and this may prove to be of interest as egg development factors represent potential therapeutic targets [47]. Other factors found included four ESTs involved in spermatogenesis and sperm motility, these factors could form future targets for chemical/therapeutic control of sheep scab as disease pathogenesis relies upon successive rounds of mite reproduction [7]. Of interest was the discovery of 23 homologues of cytochrome subunit proteins $(2.4 \%$ of the total ESTs), including a number of cytochrome P450 (CYP) factors which are one of the major factors involved in drug metabolism [48]. These may be involved in the processing of substances and compounds that are toxic to the mites, i.e. acaricidal compounds, and may therefore be important from the perspective of parasite drug resistance. The increasing dependency on macrocyclic lactone compounds to treat sheep scab combined with their continued use in the treatment of gastrointestinal nematode infections means that the future development of resistance to these compounds is inevitable. Resistance to the insecticidal compounds flumethrin and propetamphos has already been reported in $P$. ovis and resistance to ivermectin has also been recently reported [49-51]. A better understanding of the mechanisms involved in the processing of toxic compounds by $P$. ovis will allow the design of novel therapeutic targets 
and may also enable the identification of genetic markers of resistance in the mite population.

\section{KEGG pathways}

To identify signalling and metabolic pathways enriched within the $P$. ovis EST set, annotated sequences were mapped against the KEGG pathway database [52]. This analysis identified 78 enriched pathways within the current $P$. ovis transcriptome, ranging from pathways with a single member represented by a $P$. ovis EST, i.e. NGlycan biosynthesis, to the purine metabolism pathway represented by 21 individual $P$. ovis ESTs (Table 4). Figure 2 shows a representation of the mapping of the peptides inferred from the $P$. ovis transcriptome to known metabolic pathways and highlights the complex biology of this parasite. From within this map individual pathways of interest can be identified and include metabolic pathways for amino acids, lipids, energy, nucleotides and carbohydrates and also a number of pathways involved in xenobiotic biodegradation and metabolism. Of particular interest are pathways involved in amino acid metabolism, which represents 11 individual pathways; oxidative phosphorylation (the pathway in which the most individual members were identified), fatty acid and drug metabolism. Amino acids are critical components of mite biology and it is clear from the pathways highlighted here that amino acid metabolism in $P$. ovis involves a multitude of enzymes spread over many separate and interconnected pathways. The most enriched pathway identified here was that of oxidative phosphorylation. This is a metabolic pathway involved in the oxidation of nutrients to produce ATP as an energy resource [53]. We identified a number of predicted $P$. ovis enzymes involved in this pathway indicating its importance to the fundamental biology of the mite. The synthesis and metabolism of fatty acids is a crucial stage

Table 4 Top 10 enriched KEGG pathways and number of ESTs represented

\begin{tabular}{cc}
\hline KEGG Pathway & $\begin{array}{c}\text { No. of } \boldsymbol{P} \text {. ovis ESTs } \\
\text { represented }\end{array}$ \\
\hline Oxidative phosphorylation & 24 \\
Purine metabolism & 21 \\
Biosynthesis of alkaloids & 21 \\
Biosynthesis of plant hormones & 15 \\
Methane metabolism & 10 \\
Biosynthesis of phenylpropanoids & 9 \\
Valine, leucine and isoleucine & 8 \\
degradation & 7 \\
Biosynthesis of terpenoids and steroids & 7 \\
Glcerophospholipid metabolism & 6
\end{tabular}

Summary of the top 10 KEGG pathways enriched for in the $P$. ovis EST dataset. Also listed is the number of $P$. ovis ESTs represented in each pathway. in the growth and development of all parasites and the fatty acid metabolism pathway was enriched in the $P$. ovis transcriptome, with 6 potential $P$. ovis enzymes identified as being involved in this process.

Two predicted $P$. ovis enzymes identified here were a flavin-containing monooxygenase (FMO) and a uridine 5'-diphospho-glucuronosyltransferase (UDP-glucuronosyltransferase, UGT). Both enzymes are involved in the catalysis and processing of xenobiotics and are crucial for the elimination of toxic foreign chemicals and drugs from the body $[54,55]$. Pathway enrichment analysis of $P$. ovis ESTs has two distinct advantages, the first is to enable the identification of the signalling pathways involved in the basic mite biology and the second is to give greater confidence in these findings as they are based upon functional groupings of ESTs and not on single ESTs in isolation. We have described the identification of members of a number of key biological pathways in the mite and we have also described these pathways in the context of a much greater pathway, the P. ovis metabolome.

\section{Conclusions}

Here we have described the generation and analysis of 1545 unique and assembled EST sequences for the ectoparasitic mite $P$. ovis from a mixed pool of all life and sex stages. This is by far the largest such resource available for this parasite so far. The majority of these sequences (961 ESTs, 62\%) showed similarity to known genes allowing an in depth functional analysis of the $P$. ovis transcriptome. 584 ESTs (38\%) showed no similarity to known sequences, these may represent novel, as yet uncharacterised $P$. ovis transcripts and this finding highlights the current dearth of mite EST sequences available in the public databases. During this study we identified a number of important homologues, including HDM allergens and tick salivary factors which offer new insights into the underlying biology of the mite and also into the host-parasite relationship. It is anticipated that further exploration of the host:parasite relationship in sheep scab will enable the development of new methods of parasite control, i.e. identification of homologous mite allergens suitable for use as vaccine candidates and may also aid development of novel drug targets.

The generation of this EST resource will also enable the production of $P$. ovis cDNA microarrays for further analysis of gene expression in these mites and other closely related species. This resource will also allow the transcriptional analysis of different life cycles stages, i.e. egg, larva, nymphal stages and adults and analysis of the consequences of drug exposure, thus facilitating investigations into mite virulence and drug resistance mechanisms. 


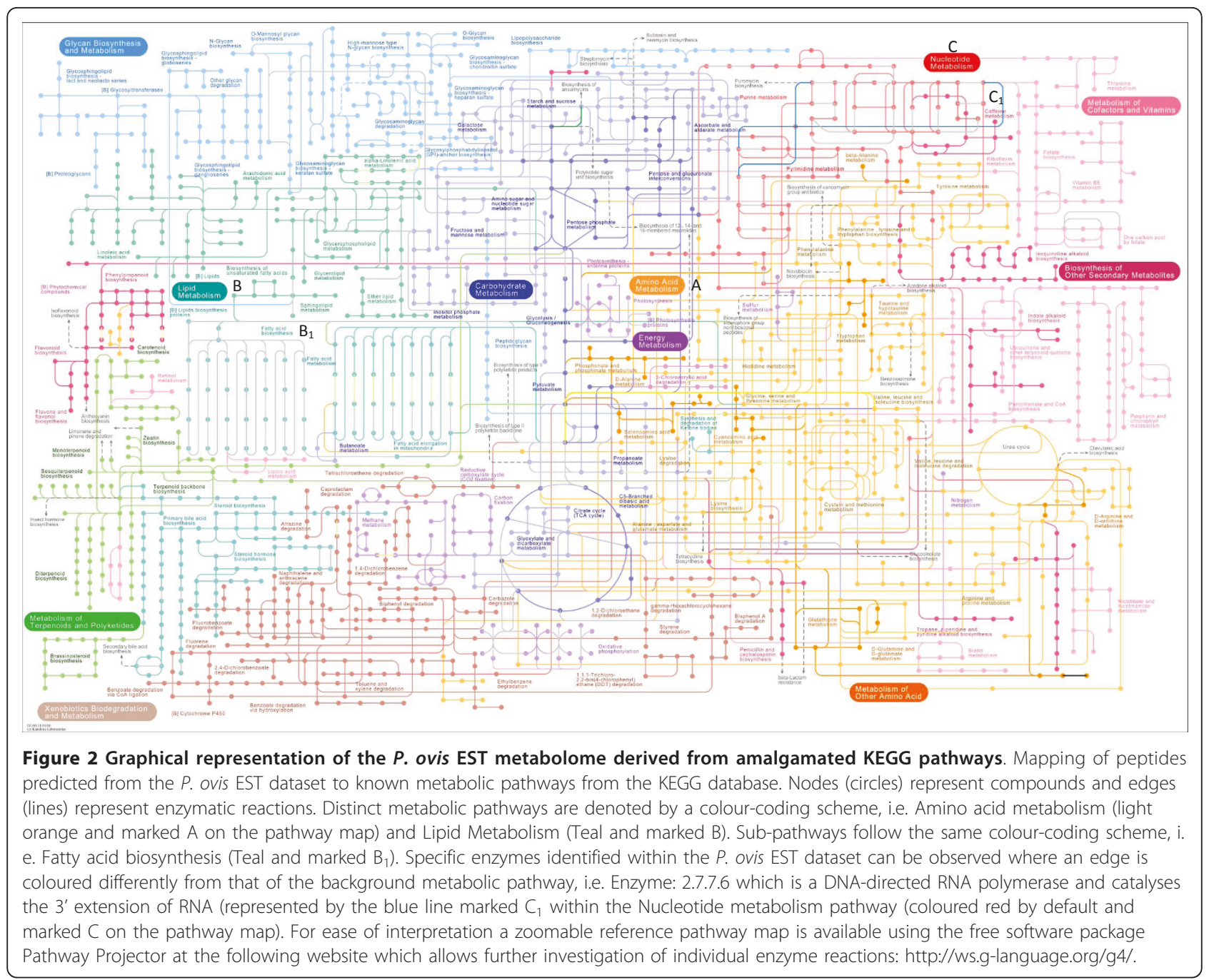

\section{Methods}

\section{$P$. ovis mite collection}

Ethical approval for this study was obtained from the Moredun Research Institute Experiments Committee and animals were monitored daily in accordance with guidelines agreed with the UK Home Office. P. ovis mites (a mixed population consisting of adults, nymphs and larvae) were harvested from infected donor animals maintained at the Moredun Research Institute as previously described [6] and care taken to remove contaminating skin debris by extensive washing. For the construction of the $P$. ovis cDNA library $800 \mathrm{mg}$ of mites were immediately snap frozen in liquid nitrogen and stored at $-80^{\circ} \mathrm{C}$ for later RNA extraction.

\section{Extraction of total RNA from $P$. ovis}

Total RNA was isolated from mites as described previously [41]. RNA samples were further purified using a Qiagen RNeasy kit, following the manufacturer's RNA cleanup protocol and on-column DNase I digestion for 15 minutes at room temperature, before elution into RNase free $\mathrm{dH}_{2} \mathrm{O}$. P. ovis RNA yield was assessed on a ND-1000 Nanodrop spectrophotometer (Thermo Scientific, UK) and RNA sample quality assessed on an Agilent Bioanalyser (Agilent, UK) and RNA Integrity Number (RIN) obtained for each sample. The RIN value is based on a ratio score of the $18 \mathrm{~s}$ and $28 \mathrm{~s}$ ribosomal RNA (rRNA) peaks, unfortunately RINs could not be obtained from the $P$. ovis RNA because, as is the case for many arthropods, the 28s rRNA species in $P$. ovis migrates with the 18s rRNA [56]. However the presence of a clean prominent peak for the 18s rRNA and no obvious degradation product indicated that the $P$. ovis RNA was of both high quality and purity.

\section{Construction of a $P$. ovis mite cDNA library}

A normalised $P$. ovis cDNA library was constructed from total RNA extracted as detailed above (Eurofins 
MWG, Germany). The cloning vector used was pBluescript II sk+ and the cloning sites were 5' EcoRI and 3' BamHI, incorporating 5' and 3' adapter sequences. The bacterial strain used was NEB 10-beta. The library titre was estimated to be $1150 \mathrm{cfu} / \mu \mathrm{l}$, the total number of clones was estimated to be 2.3 million and the average insert size was $\sim 0.9 \mathrm{~kb}$. Normalisation was carried out by denaturation and subsequent reassociation of the cDNA strands.

\section{Sequencing of clones}

The cDNA inserts of randomly selected clones were amplified by polymerase chain reaction (PCR) using M13 primers and sequence was obtained for 1574 randomly selected clones (Eurofins MWG, Germany). A further 484 P. ovis ESTs generated in a previous study by our group (accession numbers in the NCBI dbEST database from BQ834597.1 - BQ835080.1) were then added to the 1574 ESTs described above resulting in a total of 2,058 sequences for downstream analysis [14].

\section{Bioinformatical analysis}

Sequences were checked and filtered to remove low quality sequence data (EST length $<100 \mathrm{bp}$ ), vector and adaptor sequences and to mask polyA sequence using the vector tools at EMBL-EBI (http://www.ebi.ac.uk/ Tools/sss/ncbiblast/vectors.html). ESTs with an overlap of $>50$ bp were assembled into contiguous sequences "contigs" using the CAP3 sequence assembly program [57]. In order to identify homologs of the assembled $P$. ovis EST sequences, all singletons and contigs were BLAST searched against the Genbank non-redundant nucleotide and amino acid databases using the BLASTX and BLASTN algorithms within the blast2go application $[15-17,58]$. Sequences with non-significant or no BLAST score [Expectation (E) value $\leq 1.0 \mathrm{E}-4$ and/or no BLAST hit] were excluded from further analysis. E-value describes the number of hits expected by chance when searching a database of a particular size, the lower the E-value the more significant the match [58].

\section{Functional classification of $P$. ovis ESTs}

Putative biological functions and gene ontology classifications (GO) were assigned to the unique ESTs using the mapping and annotation functions in the blast2go package [15-17]. In addition ESTs were mapped to metabolic pathways and where available Enzyme Commission (EC) codes and Interproscan IDs assigned using the respective options in blast2go [15-17]. Potential signal peptide domains were identified using the SignalP 3.0 server, using both the Neural Networks and Hidden Markov Models algorithm [59] and predicted transmembrane domains were identified using the TMHMM 2.0 server (http://www.cbs.dtu.dk/services/TMHMM/) both from within the blast2go package.

\section{Acknowledgements}

This study was funded by the Department for Environment, Food and Rural Affairs (DEFRA), UK under project OD0553. The authors would like to express their gratitude to Eurofins MWG for construction of the normalized $P$. ovis CDNA library and for sequencing of the CDNA clones. We would also like to thank Alex Lainson \& Raja Yaga of the Moredun Bioinformatics Division for their bioinformatics support and BLAST analysis and the Moredun Clinical

Division for their continued help and expertise.

\section{Authors' contributions}

STGB designed the study, performed and processed samples and data analysis and wrote the manuscript. AJN participated in the study design and data analysis and helped prepare the manuscript. FK generated a $P$. ovis cDNA library, helped with data interpretation and preparation of the manuscript. JFH conceived and designed the study, participated in the analysis and helped prepare the manuscript. All authors have read and approved the manuscript

\section{Competing interests}

The authors declare that they have no competing interests.

Received: 14 June 2011 Accepted: 22 July 2011 Published: 22 July 2011

\section{References}

1. Jones J, Jenkins T, Webb L, Davies A, Bates P: Psoroptic mange in cattle in south Wales. Vet Rec 2008, 162:460.

2. Kirkwood AC: History, biology and control of sheep scab. Parasitol Today 1986, 2:302-307.

3. Nisbet AJ, Huntley JF: Progress and opportunities in the development of vaccines against mites, fleas and myiasis-causing flies of veterinary importance. Parasite Immunol 2006, 28:165-172.

4. DeLoach JR, Wright FC: Ingestion of rabbit erythrocytes containing $51 \mathrm{Cr}$ labeled hemoglobin by Psoroptes spp. (Acari: Psoroptidae) that originated on cattle, mountain sheep, or rabbits. J Med Entomol 1981, 18:345-348.

5. Mathieson BR, Lehane MJ: Ultrastructure of the alimentary canal of the sheep scab mite, Psoroptes ovis (Acari: Psoroptidae). Vet Parasitol 2002, 104:151-166.

6. Burgess ST, Frew D, Nunn F, Watkins CA, McNeilly TN, Nisbet AJ, Huntley JF: Transcriptomic analysis of the temporal host response to skin infestation with the ectoparasitic mite Psoroptes ovis. BMC Genomics 2010, 11:624.

7. van den Broek AH, Huntley JF: Sheep scab: the disease, pathogenesis and control. J Comp Pathol 2003, 128:79-91.

8. Hamilton KA, Nisbet AJ, Lehane MJ, Taylor MA, Billingsley PF: A physiological and biochemical model for digestion in the ectoparasitic mite, Psoroptes ovis (Acari: Psoroptidae). Int J Parasitol 2003, 33:773-785.

9. Sinclair AN, Filan SJ: Confirmation of degenerative effects on psoroptic mites from scab lesions. Vet Rec 1991, 129:492.

10. Thomas WR, Smith W: House-dust-mite allergens. Allergy 1998, 53:821-832.

11. Bates P: Differences between primary and secondary infestations with the sheep scab mite, Psoroptes ovis. Vet Rec 2000, 146:528-529.

12. Smith WD, Bates P, Pettit DM, Van Den Broek A, Taylor MA: Attempts to immunize sheep against the scab mite, Psoroptes ovis. Parasite Immunol 2002, 24:303-310.

13. Smith WD, van den Broek A, Huntley J, Pettit D, Machell J, Miller HR, Bates P, Taylor M: Approaches to vaccines for Psoroptes ovis (sheep scab). Res Vet Sci 2001, 70:87-91.

14. Kenyon F, Welsh M, Parkinson J, Whitton C, Blaxter ML, Knox DP: Expressed sequence tag survey of gene expression in the scab mite Psoroptes ovis-allergens, proteases and free-radical scavengers. Parasitology 2003, 126:451-460

15. Conesa A, Gotz S: Blast2GO: A comprehensive suite for functional analysis in plant genomics. Int J Plant Genomics 2008, 2008:619832.

16. Conesa A, Gotz S, Garcia-Gomez JM, Terol J, Talon M, Robles M: Blast2GO: a universal tool for annotation, visualization and analysis in functional genomics research. Bioinformatics 2005, 21:3674-3676.

17. Gotz S, Garcia-Gomez JM, Terol J, Williams TD, Nagaraj SH, Nueda MJ, Robles M, Talon M, Dopazo J, Conesa A: High-throughput functional annotation and data mining with the Blast2GO suite. Nucleic Acids Res 2008, 36:3420-3435.

18. Zdobnov EM, Apweiler R: InterProScan-an integration platform for the signature-recognition methods in InterPro. Bioinformatics 2001, 17:847-848. 
19. Kelly RJ, Vincent DE, Friedberg I: IPRStats: visualization of the functional potential of an InterProScan run. BMC Bioinformatics 2010, 11(Suppl 12): S13..

20. Emanuelsson $\mathrm{O}$, Brunak $\mathrm{S}$, von Heijne $\mathrm{G}$, Nielsen $\mathrm{H}$ : Locating proteins in the cell using TargetP, SignalP and related tools. Nat Protoc 2007, 2:953-971.

21. Krogh A, Larsson B, von Heijne G, Sonnhammer EL: Predicting transmembrane protein topology with a hidden Markov model: application to complete genomes. J Mol Biol 2001, 305:567-580.

22. Hegde RS, Bernstein HD: The surprising complexity of signal sequences. Trends Biochem Sci 2006, 31:563-571.

23. Park GM, Lee SM, Lee IY, Ree HI, Kim KS, Hong CS, Yong TS: Localization of a major allergen, Der $\mathrm{p} \mathrm{2}$, in the gut and faecal pellets of Dermatophagoides pteronyssinus. Clin Exp Allergy 2000, 30:1293-1297.

24. Preiss T, M WH: Starting the protein synthesis machine: eukaryotic translation initiation. Bioessays 2003, 25:1201-1211.

25. Kenyon F, Knox D: The proteinases of Psoroptes ovis, the sheep scab mite-their diversity and substrate specificity. Vet Parasitol 2002, 105:317-325

26. Clara RO, Soares TS, Torquato RJ, Lima CA, Watanabe RO, Barros NM, Carmona AK, Masuda A, Vaz ISJ, Tanaka AS: Boophilus microplus cathepsin L-like (BmCL1) cysteine protease: Specificity study using a peptide phage display library. Vet Parasitol 2011.

27. Kato T, Takai T, Mitsuishi K, Okumura K, Ogawa H: Cystatin A inhibits IL-8 production by keratinocytes stimulated with Der $\mathrm{p} 1$ and Der $\mathrm{f} 1$ : biochemical skin barrier against mite cysteine proteases. J Allergy Clin Immunol 2005, 116:169-176.

28. Chuang CY, Chang CH, Huang YL: Thioredoxin mediates remodeling factors of human bronchial epithelial cells upon interaction with house dust mite-stimulated eosinophils. Inhal Toxicol 2009, 21:153-167.

29. Karabulut AB, Atambay M, Karaman U, Kilic E, Yazar S, Saraymen R, Daldal N House dust-mites: effect on antioxidant enzyme activities. Med Sci Monit 2006, 12:CR378-381.

30. Mounsey KE, Pasay CJ, Arlian LG, Morgan MS, Holt DC, Currie BJ, Walton SF, McCarthy JS: Increased transcription of Glutathione S-transferases in acaricide exposed scabies mites. Parasit Vectors 2010, 3:43.

31. Armstrong RN: Glutathione S-transferases: reaction mechanism, structure, and function. Chem Res Toxicol 1991, 4:131-140.

32. O'Neill GM, Donovan GR, Baldo BA: Cloning and characterization of a major allergen of the house dust mite, Dermatophagoides pteronyssinus, homologous with glutathione S-transferase. Biochim Biophys Acta 1994, 1219:521-528.

33. Dougall A, Holt DC, Fischer K, Currie BJ, Kemp DJ, Walton SF: Identification and characterization of Sarcoptes scabiei and Dermatophagoides pteronyssinus glutathione S-transferases: implication as a potential major allergen in crusted scabies. Am J Trop Med Hyg 2005, 73:977-984

34. Sarrias MR, Gronlund J, Padilla O, Madsen J, Holmskov U, Lozano F: The Scavenger Receptor Cysteine-Rich (SRCR) domain: an ancient and highly conserved protein module of the innate immune system. Crit Rev Immunol 2004, 24:1-37.

35. Sasaki SD, de Lima CA, Lovato DV, Juliano MA, Torquato RJ, Tanaka AS: BmSI-7, a novel subtilisin inhibitor from Boophilus microplus, with activity toward $\operatorname{Pr} 1$ proteases from the fungus Metarhizium anisopliae. Exp Parasitol 2008, 118:214-220.

36. Ovaere $P$, Lippens $S$, Vandenabeele $P$, Declercq $W$ : The emerging roles of serine protease cascades in the epidermis. Trends Biochem Sci 2009 , 34:453-463.

37. Lee AJ, Machell J, Van Den Broek AH, Nisbet AJ, Miller HR, Isaac RE, Huntley JF: Identification of an antigen from the sheep scab mite, Psoroptes ovis, homologous with house dust mite group I allergens. Parasite Immunol 2002, 24:413-422.

38. Nisbet AJ, Halliday AM, Parker L, Smith WD, Kenyon F, Knox DP, Huntley JF: Psoroptes ovis: identification of vaccine candidates by immunoscreening. Exp Parasitol 2008, 120:194-199.

39. Huntley JF, Machell J, Nisbet AJ, Van den Broek A, Chua KY, Cheong N, Hales BJ, Thomas WR: Identification of tropomyosin, paramyosin and apolipophorin/vitellogenin as three major allergens of the sheep scab mite, Psoroptes ovis. Parasite Immunol 2004, 26:335-342.

40. Weghofer M, Dall'Antonia Y, Grote M, Stocklinger A, Kneidinger M, Balic N, Krauth MT, Fernandez-Caldas E, Thomas WR, van Hage M, et al:
Characterization of Der p 21, a new important allergen derived from the gut of house dust mites. Allergy 2008, 63:758-767.

41. Maruo K, Akaike T, Ono T, Okamoto T, Maeda H: Generation of anaphylatoxins through proteolytic processing of $\mathrm{C} 3$ and $\mathrm{C} 5$ by house dust mite protease. J Allergy Clin Immunol 1997, 100:253-260.

42. Hong M, Kwon JY, Shim J, Lee J: Differential hypoxia response of hsp-16 genes in the nematode. J Mol Biol 2004, 344:369-381.

43. Strayer A, Wu Z, Christen Y, Link CD, Luo Y: Expression of the small heatshock protein Hsp16-2 in Caenorhabditis elegans is suppressed by Ginkgo biloba extract EGb 761. FASEB J 2003, 17:2305-2307.

44. Richards AG, Richards PA: The peritrophic membranes of insects. Annu Rev Entomol 1977, 22:219-240.

45. Lue H, Kleemann R, Calandra T, Roger T, Bernhagen J: Macrophage migration inhibitory factor (MIF): mechanisms of action and role in disease. Microbes Infect 2002, 4:449-460

46. Dobson SE, Augustijn KD, Brannigan JA, Schnick C, Janse CJ, Dodson EJ, Waters AP, Wilkinson AJ: The crystal structures of macrophage migration inhibitory factor from Plasmodium falciparum and Plasmodium berghei. Protein Sci 2009, 18:2578-2591.

47. Abouel-Nour MF, Lotfy M, Attallah AM, Doughty BL: Schistosoma mansoni major egg antigen Smp40: molecular modeling and potential immunoreactivity for anti-pathology vaccine development. Mem Inst Oswaldo Cruz 2006, 101:365-372.

48. Danielson PB: The cytochrome P450 superfamily: biochemistry, evolution and drug metabolism in humans. Curr Drug Metab 2002, 3:561-597.

49. Synge BA, Bates PG, Clark AM, Stephen FB: Apparent resistance of $P$ ovis to flumethrin. Vet Rec 1995, 137:51.

50. Clark AM, Stephen FB, Cawley GD, Bellworthy SJ, Groves BA: Resistance of the sheep scab mite Psoroptes ovis to propetamphos. Vet Rec 1996, 139:451

51. Lekimme M, Farnir F, Marechal F, Losson B: Failure of injectable ivermectin to control psoroptic mange in cattle. Vet Rec 2010, 167:575-576.

52. Kanehisa M, Goto S: KEGG: kyoto encyclopedia of genes and genomes. Nucleic Acids Res 2000, 28:27-30.

53. Gautheron DC: Mitochondrial oxidative phosphorylation and respiratory chain: review. J Inherit Metab Dis 1984, 7(Suppl 1):57-61.

54. Cashman JR: Structural and catalytic properties of the mammalian flavincontaining monooxygenase. Chem Res Toxicol 1995, 8:166-181.

55. King CD, Rios GR, Green MD, Tephly TR: UDP-glucuronosyltransferases. Curr Drug Metab 2000, 1:143-161.

56. Winnebeck EC, Millar CD, Warman GR: Why does insect RNA look degraded? J Insect Sci 2010, 10:159.

57. Huang X, Madan A: CAP3: A DNA sequence assembly program. Genome Res 1999, 9:868-877.

58. Altschul SF, Gish W, Miller W, Myers EW, Lipman DJ: Basic local alignment search tool. J Mol Biol 1990, 215:403-410.

59. Bendtsen JD, Nielsen H, von Heijne G, Brunak S: Improved prediction of signal peptides: SignalP 3.0. J Mol Biol 2004, 340:783-795.

\section{doi:10.1186/1756-3305-4-145}

Cite this article as: Burgess et al:: Generation, analysis and functional annotation of expressed sequence tags from the ectoparasitic mite Psoroptes ovis. Parasites \& Vectors 2011 4:145.

\section{Submit your next manuscript to BioMed Central and take full advantage of:}

- Convenient online submission

- Thorough peer review

- No space constraints or color figure charges

- Immediate publication on acceptance

- Inclusion in PubMed, CAS, Scopus and Google Scholar

- Research which is freely available for redistribution 